\title{
EchoGéo
}

\section{Rues en parallèle, une étude comparative entre Shanghai et Paris}

Antoine Brès, Jean-François Cuenot et Thierry Sanjuan

\section{Q OpenEdition}

\section{Journals}

Édition électronique

URL : https://journals.openedition.org/echogeo/11902

DOI : 10.4000/echogeo. 11902

ISSN : 1963-1197

\section{Éditeur}

Pôle de recherche pour l'organisation et la diffusion de l'information géographique (CNRS UMR 8586)

\section{Référence électronique}

Antoine Brès, Jean-François Cuenot et Thierry Sanjuan, «Rues en parallèle, une étude comparative entre Shanghai et Paris », EchoGéo [En ligne], 12 | 2010, mis en ligne le 31 mai 2010, consulté le 31 juillet 2021. URL : http://journals.openedition.org/echogeo/11902 ; DOI : https://doi.org/10.4000/ echogeo.11902

Ce document a été généré automatiquement le 31 juillet 2021.

EchoGéo est mis à disposition selon les termes de la licence Creative Commons Attribution - Pas d'Utilisation Commerciale - Pas de Modification 4.0 International (CC BY-NC-ND) 


\title{
Rues en parallèle, une étude comparative entre Shanghai et Paris
}

\author{
Antoine Brès, Jean-François Cuenot et Thierry Sanjuan
}

1 Parler aujourd'hui de la rue d'une grande ville chinoise, c'est à la fois parler de toutes les rues des grandes métropoles contemporaines et parler de la Chine. Les gravures anciennes comme la "Pure Brightness River Chart », peinte durant les dynasties Ming et Qing, et achevée au début du XVIII siècle, décrivent avec beaucoup de minutie, en perspective cavalière plongeante - sur plus de 11 mètres de long -, les activités qui se déploient pour la plus grande part dans l'espace d'une rue reliant les portes d'un palais au rempart, et au-delà à la campagne et au bord du fleuve. Tout le long de son tracé, et de part et d'autre de ses deux rives, on observe une multitude de saynettes qui mettent en relation le passant et le résidant, le commerçant ambulant ou d'échoppe, le mendiant, le saltimbanque, le cavalier, le marcheur ou la voiture à cheval, le chariot... et où se combinent de part et d'autre des façades espace public et intimité à travers différents seuils suivant le statut des riverains : cour, entrée, jardin...

Une fois dépassé tout ce qui caractérise aussi bien la civilisation chinoise que la période historique décrite ici, costumes et architecture notamment, on retrouve tous les ingrédients de ce qui fait la rue, sa vocation première de combiner établissement et mouvement, pour reprendre les catégories d'Idelfonso Cerda (Cerda I., 1867, 1979), et que décrivent aussi bien dans un autre lieu et à une autre époque les gravures de Londres de Gustave Doré par exemple.

2 Aujourd'hui, pour qui emprunte la rue d'une métropole chinoise en plein bouleversement, et qui connaît un processus accéléré de globalisation, se pose la question de ce qui fait sa permanence et sa singularité, notamment les différences et les similitudes que l'on peut observer entre cette rue en Chine et son équivalent de par le monde. La comparaison de deux rues péricentrales et commerçantes, l'une shanghaienne - la rue du Sichuan (Nord, dans son tronçon septentrional) - et l'autre parisienne - la rue du faubourg Saint-Antoine - de vocation et de situation similaires, nous aide à répondre à ces questions. Cette mise en parallèle, à l'ambition limitée d'une démarche comparative, a été menée à partir d'observations et d'analyses, et complétée 
par des enquêtes et des entretiens conduits par les étudiants de l'Université Paris 1 Panthéon-Sorbonne à la faveur de différents travaux, dont certains réalisés dans le cadre d'une collaboration entre l'UMR PRODIG et le CAUP de l'Université Tongji à Shanghai (ateliers, séminaires franco-chinois et mémoires dont les figures présentées sont issues).

Illustrations 1 et 2 - Morphologie du bâti et des espaces libres

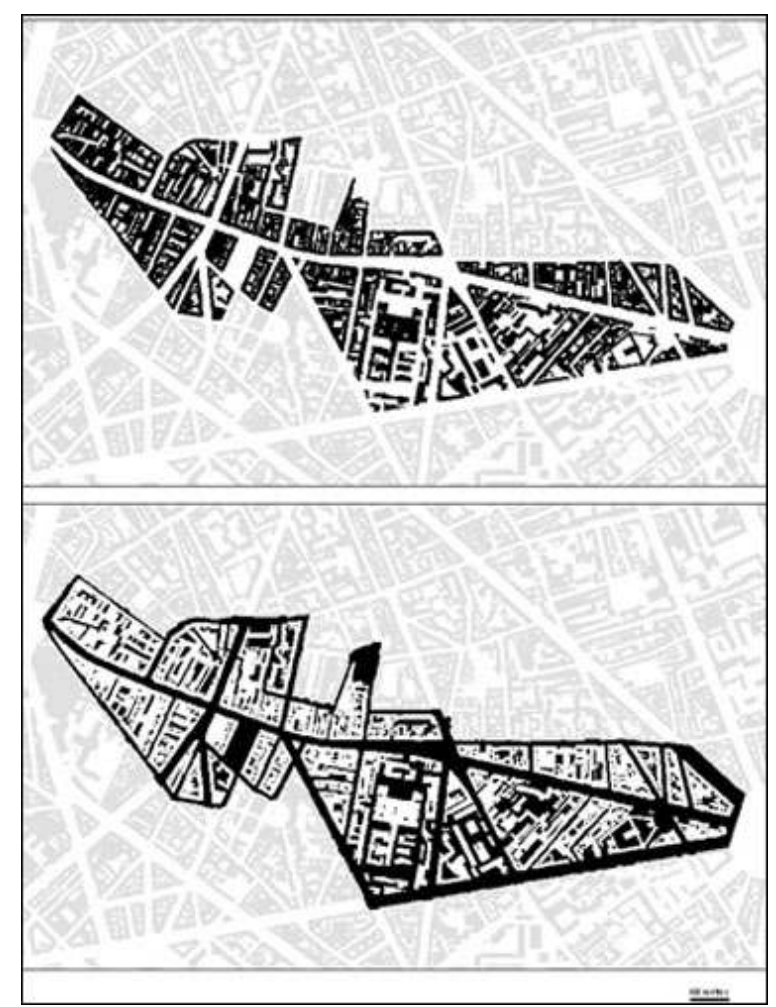

Rue du faubourg Saint-Antoine 


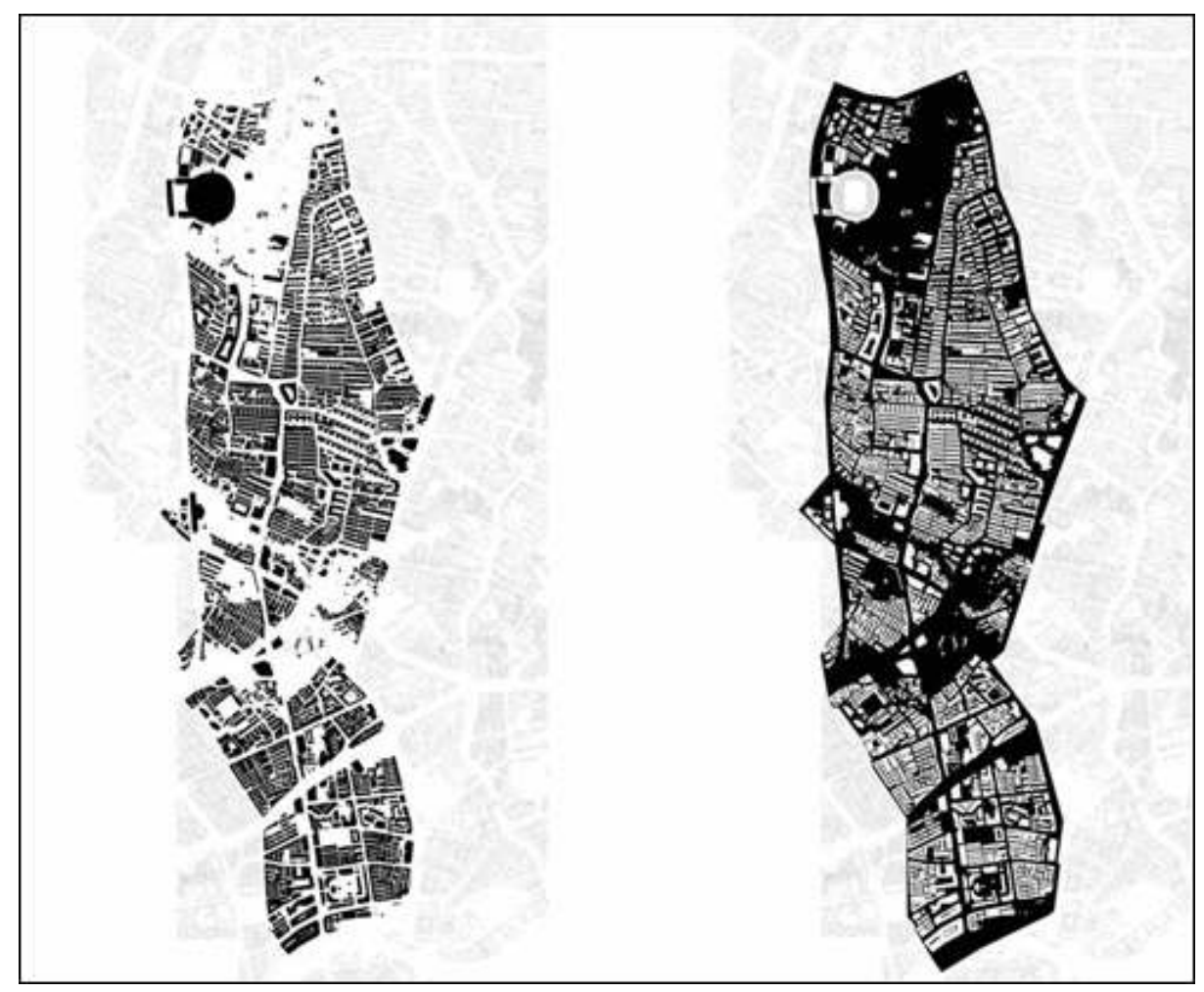

Rue du Sichuan (Nord)

3 Après une rapide mise en situation préalable de ces deux rues aux plans historique et urbain, on s'intéressera en premier lieu à leur morphologie générale, aussi bien celle des espaces libres de statut public et privé que du bâti riverain - les typologies résidentielle et commerciale -, et au paysage urbain qu'ils définissent conjointement. On abordera leurs mutations récentes, notamment la part allouée au patrimoine et à la modernité dans le renouvellement urbain du bâti riverain.

On s'intéressera ensuite à la façon dont les différentes pratiques s'organisent aussi bien dans l'espace public que constitue l'emprise de la rue elle-même que dans l'épaisseur de ses rives, les fonctions urbaines qu'elles accueillent - résidentielles et commerciales plus particulièrement - et les échelles territoriales auxquelles elles se référent - locale et métropolitaine.

5 Enfin, la synthèse de ces observations permettra de pointer les transformations que connaissent ces deux rues et notamment la gentrification qui les caractérise sur plusieurs aspects. On relèvera en particulier le rôle essentiel du commerce dans les évolutions des pratiques de ces deux rues et qui les différencie sans doute aujourd'hui. Nous verrons si «la rue en se perpétuant garantit l'unité dans la diversité et le changement » comme l'affirme J.-L. Gourdon (2001), en particulier dans le cas chinois étudié ici.

\section{Des rues en situation: deux radiales aux vocations similaires}

6 La rue du Sichuan emprunte sur 3,7 km un tracé historique nord-sud qui s'est affirmé au XIXe siècle à l'époque des concessions étrangères comme axe majeur de liaison entre 
la rivière Suzhou et la gare de chemin de fer Songhu, et de développement commercial entre ces deux polarités urbaines. En 1902, la rue est prolongée jusqu'au nouveau parc de Lu Xun pour le relier au centre-ville. À la suite, à partir de 1919, des projets d'élargissement et d'homogénéisation de la rue ont été lancés mais n'ont jamais été menés à terme en raison des événements politiques qui sont survenus à partir des années 1930 - occupation japonaise, révolution, réformes et « ouverture ».

7 Elle présente dès cette époque une grande mixité sociale et fonctionnelle. Ainsi, en 1930 , on $y$ trouvait 32 cinémas, tandis que 47 sociétés de production cinématographique s'étaient implantées dans le district de Hongkou. Alors que la population de la concession internationale, au sein de laquelle s'inscrivait la rue du Sichuan (Nord), s'élevait à 840000 habitants incluant Britanniques et Américains en 1925, elle atteignait 1140000 habitants en 1934, issus pour beaucoup de l'immigration intérieure, en provenance des provinces des Jiangsu, Zhejiang et Guangdong. Ce brassage de populations chinoises et étrangères s'intensifie encore à partir de 1937 sous l'occupation japonaise, dont certains ressortissants s'installent dans ce même district.

En 1947, la rue du Sichuan s'est considérablement développée et se présente comme une artère prospère et réputée pour son animation et ses commerces. Aujourd'hui, sa vocation commerciale est réaffirmée aussi bien à l'échelle du quartier et du district qu'à l'échelle métropolitaine, comme l'une des trois grandes rues commerçantes de Shanghai avec les rues de Huaihai (Centre) et de Nankin.

Photographie 1 - La rue du Sichuan

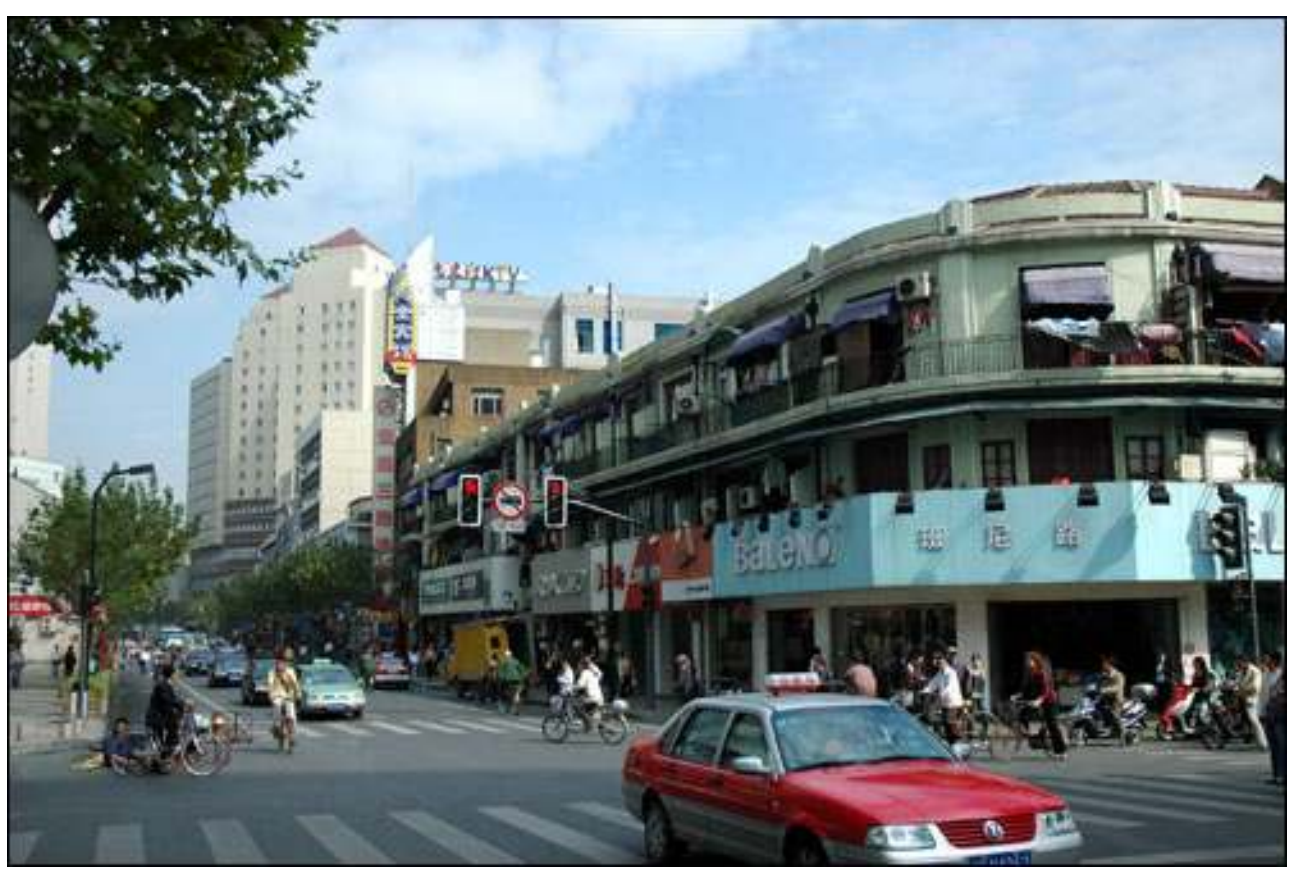




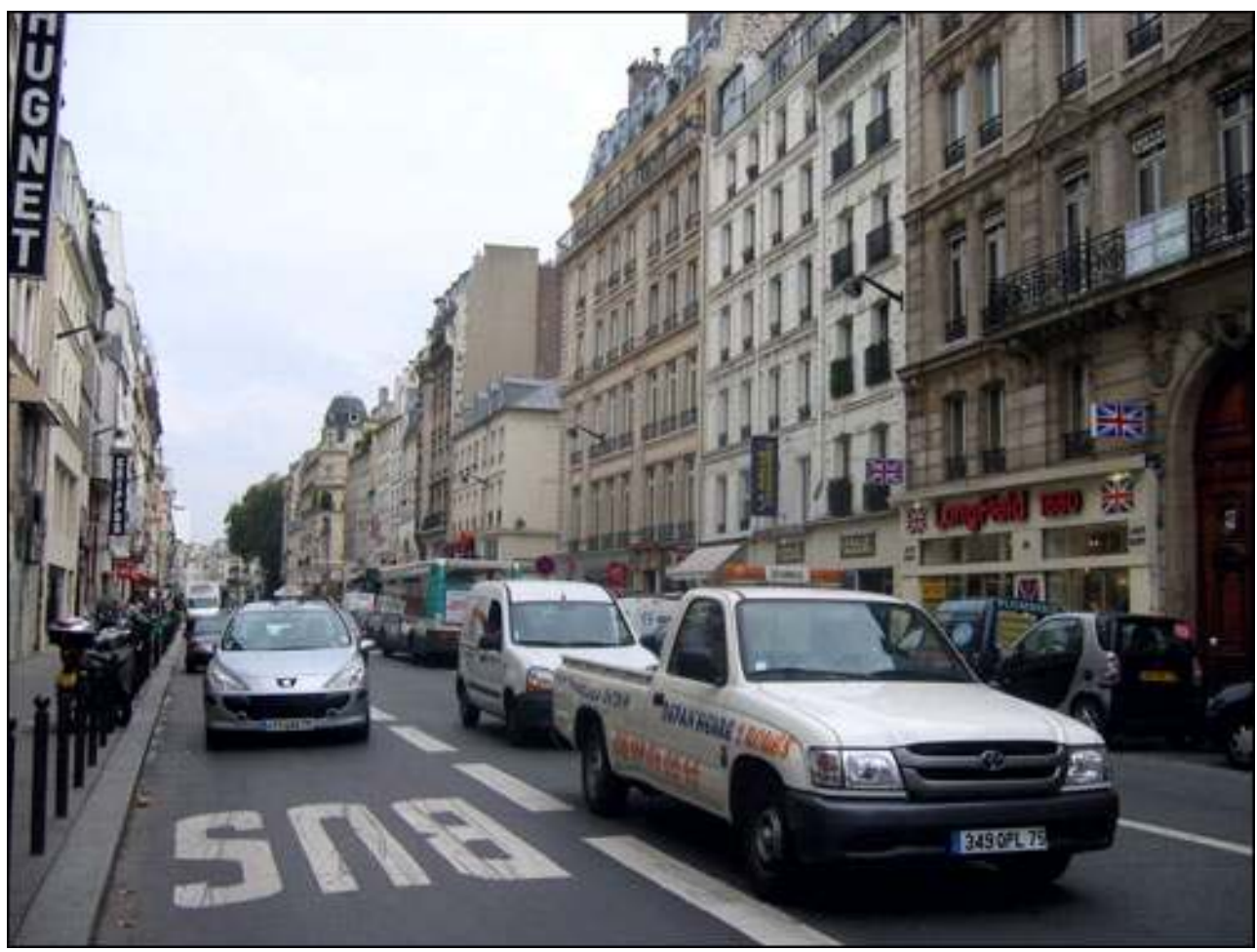

La rue du faubourg Saint-Antoine suit sur 1,8 km un tracé médiéval qui est aujourd'hui borné par deux lieux monumentaux, les places de la Bastille et de la Nation, anciennement place du Trône. L'urbanisation de la rue elle-même a précédé celle du faubourg qui porte son nom et qui ne s'est vraiment développé qu'à partir du XVIII $^{\mathrm{e}}$ siècle avec le recul des limites de Paris jusqu'aux barrières des fermiers généraux et le développement de multiples activités dans l'épaisseur des îlots qui bordaient la voie : faïencerie, métallurgie, ébénisterie... On observe ainsi à partir du milieu du $\mathrm{XIX}^{\mathrm{e}}$ siècle un processus de renouvellement urbain à l'échelle de la parcelle, qui est à l'origine de sa configuration actuelle : une organisation du bâti en profondeur autour de cours et de passage derrière des façades souvent plus anciennes.

10 Les transformations haussmanniennes du faubourg ont épargné la rue elle-même, tout en modifiant considérablement le quartier qui l'environnait. Le square Trousseau a été aménagé en 1904 à la faveur de la réalisation d'un lotissement résidentiel. Les projets «hygiénistes", puis "modernes", conçus dans la première moitié du XX $\mathrm{XX}^{\mathrm{e}}$ siècle auraient conduit à la destruction presque complète de la rue, si avaient été mis en œuvre son élargissement à 65 mètres et la résorption des "îlots insalubres » qui la bordaient.

11 Le déclin économique des activités artisanales et leur lente disparition au profit d'opérations résidentielles à partir du milieu du $\mathrm{XX}^{\mathrm{e}}$ siècle ont commencé à remettre en cause la mixité qui caractérisait le faubourg. En réponse à cette situation, un périmètre d'étude a été institué en 1994 sur 75 ha, puis étendu à 85 ha, et un ensemble de mesures et de dispositifs ont été mis en place qui prenaient en compte tous les aspects de la vie urbaine : modification du plan d'occupation des sols (POS) adoptée en 1998; mise en place d'une opération programmée d'amélioration de l'habitat (OPAH), qui a permis le maintien sur place de populations aux revenus modestes grâce à l'encadrement des 
loyers; démarche de concertation dans laquelle les associations de quartier se sont impliquées ; sauvegarde des activités artisanales ; mise en valeur des espaces publics...

Les règles du POS ont été adaptées à l'identité particulière du faubourg "sans figer excessivement la situation » en privilégiant quatre aspects de sa morphologie :

- son paysage, en adaptant le gabarit du bâti riverain, avec notamment un alignement presque systématique quelle que soit la largeur de la rue, en dérogation avec le « droit commun » parisien, qui imposait traditionnellement un prospect du bâti en proportion de cette largeur;

- l'organisation du bâti autour des cours et passages en retrait de la rue, et dont on a pu constater qu'elle présentait une grande capacité d'adaptation aux différents usages avec notamment une imbrication des domaines privé et public ;

- la mixité des fonctions en adaptant la hauteur des rez-de-chaussée aux activités et en prescrivant un coefficient d'occupation du sol (COS) différencié entre logement et activité suivant leur situation dans le bâtiment ;

- la protection des éléments les plus remarquables de cette « architecture du quotidien » - on ne trouve en effet aucun véritable monument le long de la rue -, et qui a porté en particulier sur 150 parcelles et 45 cours.

13 Aujourd'hui, malgré les transformations qu'elle a connues, notamment la gentrification de sa population, la rue témoigne encore d'une mixité urbaine remarquable.

\section{Morphologie et paysage urbain : les enjeux du patrimoine}

La période de développement majeur de ces deux rues se situe autour de la seconde moitié du XIX siècle - la «première mondialisation »- et du début du XXe siècle, avec une légère antériorité pour la rue du faubourg Saint-Antoine. Aujourd'hui de cette période, chacune de ces rues a gardé un patrimoine important.

Ce patrimoine est très majoritairement préservé dans le cas de la rue du faubourg Saint-Antoine. Les quelques opérations ponctuelles de rénovation ont été réalisées dans la profondeur des îlots et n'ont pas modifié la morphologie générale de la rue. Son gabarit présente une grande homogénéité et la continuité du bâti subsiste presque totalement.

Lue du Sichuan (Nord) présente un aspect beaucoup plus hétérogène et tire sa diversité d'une typologie résidentielle très directement issue des différentes phases de transformation politique qu'a connues Shanghai, et plus généralement la Chine, tout au long du XXe siècle : courées (lilong) de l'époque des « concessions », puis de l'occupation japonaise; ensembles résidentiels constitués de «barres» de l'époque maoïste; grandes opérations réalisées depuis le lancement des "réformes et de l'ouverture " dans les années 1980.

Sa mutation actuelle témoigne de la politique de rénovation ambitieuse et volontaire menée par le district de Hongkou, et qui paraît particulièrement brutale à l'observateur européen. La construction-destruction accélérée d'un grand nombre d'îlots hérités a un impact global sur la rue : plus de $800000 \mathrm{~m}^{2}$ de surface commerciale ont été réalisés ou sont projetés, soit plus de trois fois ce qui existait auparavant. "Les caractéristiques architecturales, historiques et sociales du bâti ancien chinois disparaissent au profit d'une spécialisation fonctionnelle de l'espace urbain » (Ged F., 2000). A titre d'exemple, 
l'élargissement de la rue Haining, qui coupe la rue du Sichuan (Nord) à mi-parcours, a entraîné la démolition de bâtiments d'un grand intérêt architectural, social et culturel, comme par exemple le plus ancien cinéma de Shanghai.

Illustration 3 - Typologie du bâti : rue du Sichuan (Nord)

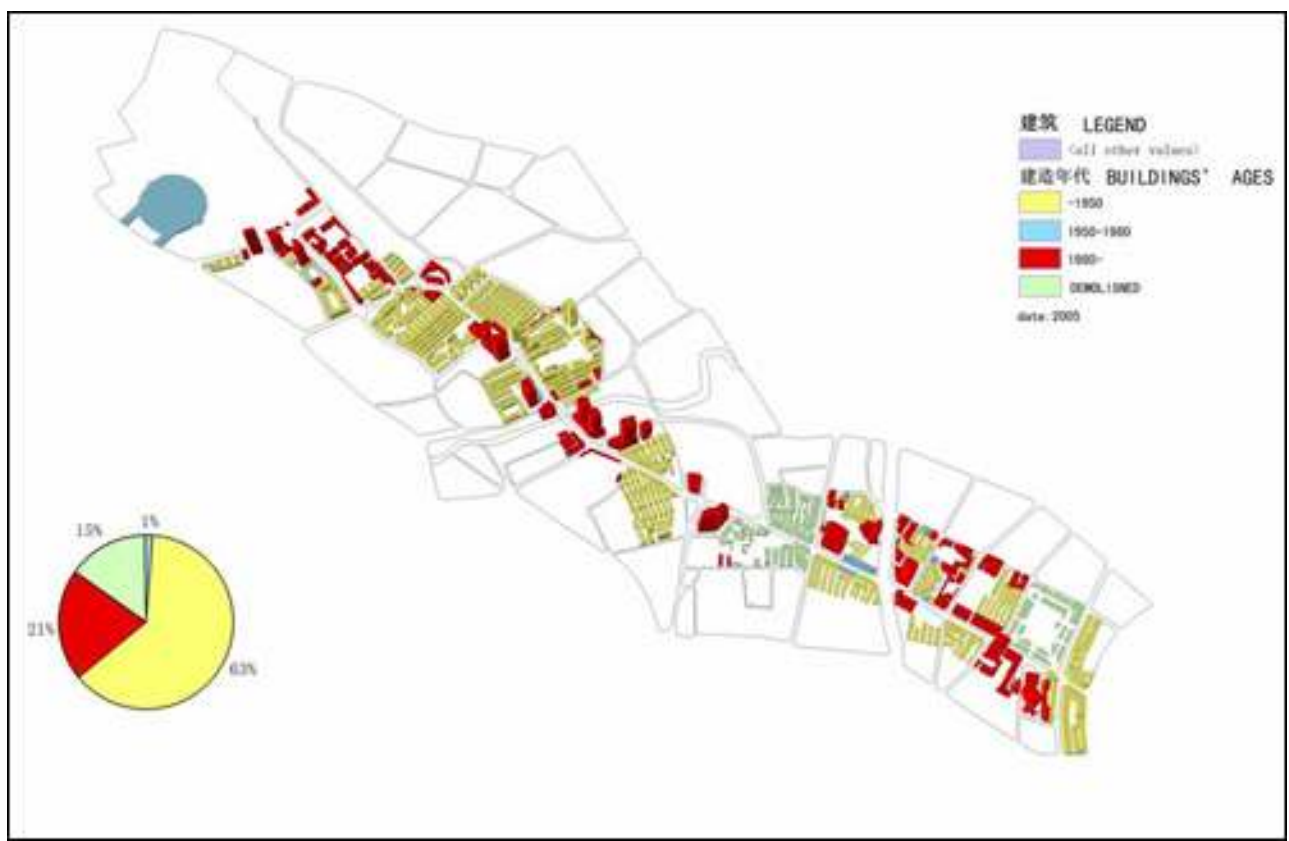

Cependant, une prise de conscience récente de l'intérêt que le patrimoine représente pour l'image et le statut métropolitains de Shanghai a conduit les autorités à protéger certains secteurs historiques comme certaines courées ou le secteur de la rue Duolun à laquelle donne accès la rue du Sichuan (Nord). 
Photographie 3 - Patrimoine urbain, rue du Sichuan

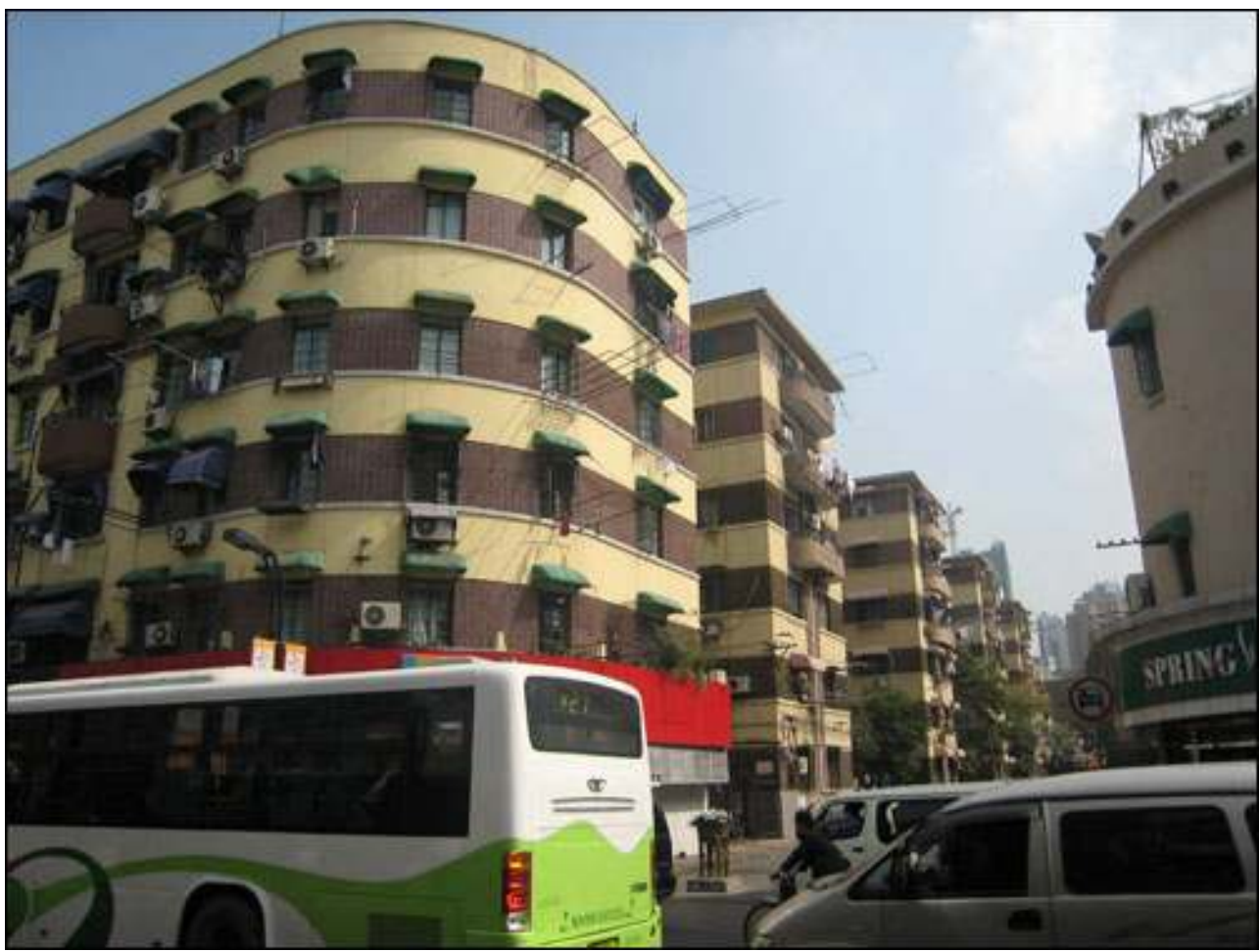

De leur patrimoine urbain, ces deux rues conservent des morphologies héritées très spécifiques qui présentent pour chacune d'elle, mais de manière différenciée, des caractéristiques de profondeur et de porosité des «territoires de la voie » (îlots et parcelles desservies par la rue): les ruelles des courées shanghaiennes et les cours artisanales parisiennes, avec une différence importante quant à la dimension des îlots et au maillage des voies. La grande taille des îlots shanghaiens est compensée par un réseau capillaire de dessertes internes, piétonnières pour l'essentiel, qui crée des liaisons d'une rue à l'autre. Elle a cependant rendu possible aujourd'hui la réalisation de vastes opérations de rénovation urbaine au détriment de ce patrimoine urbain original. La petite taille de l'îlot parisien, la prégnance du parcellaire et le morcellement de la propriété foncière ont limité l'ampleur de cette porosité, qui ne permet que très rarement des liaisons au travers de l'îlot. 
Illustration 4 - Profondeur des îlots urbains

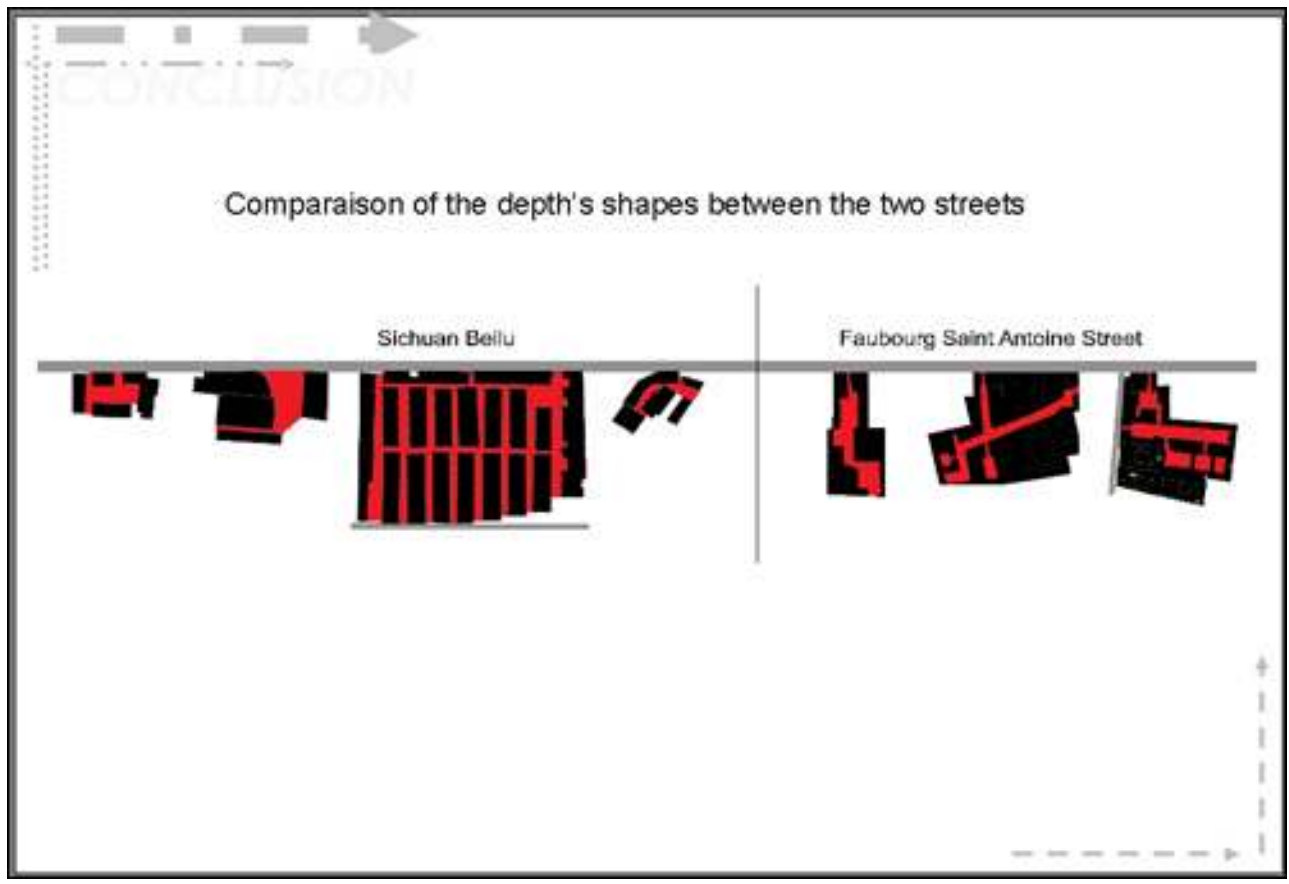

Croquis de gauche : rue du Sichuan (Nord) - croquis de droite : rue du faubourg Saint-Antoine

Photographie 4 - Passage, rue du Sichuan

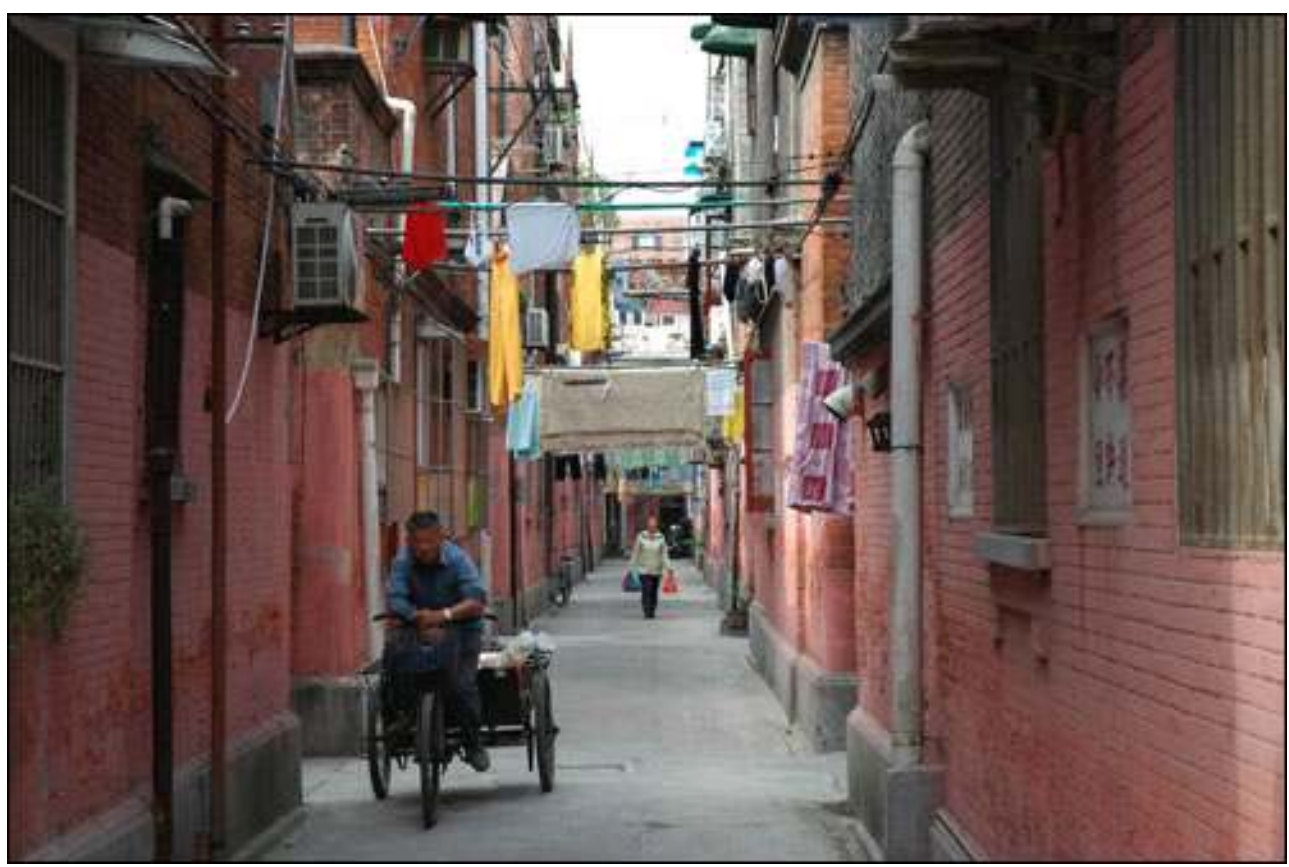




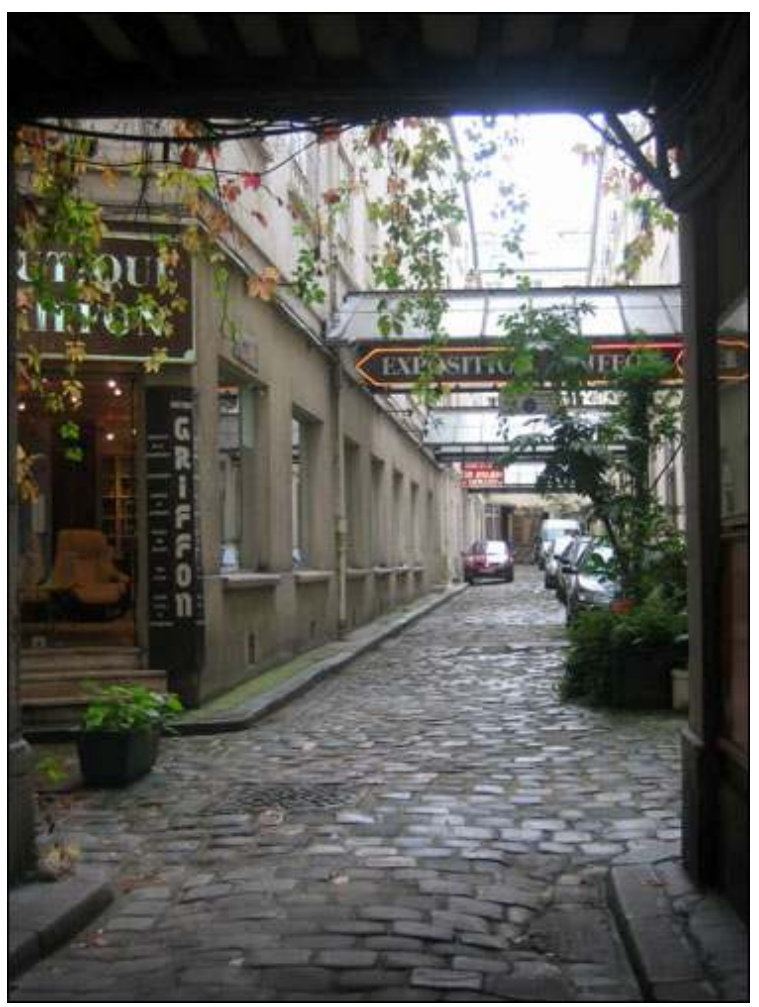

Ces deux rues conservent également de leur époque de référence des lieux de loisirs spécifiques : le square Trousseau cité en rive sud de la rue du faubourg Saint-Antoine, le parc Lu Xun à l'extrémité nord de la rue du Sichuan (Nord) et plus récemment le parc du Sichuan aménagé en 2004 dans le cadre d'une vaste opération immobilière.

\section{Pratiques résidentielles : des processus de gentrification différenciés}

Les 11 opérations de grande ampleur qui ont vu le jour depuis 2005 le long de la rue du Sichuan (Nord) ont été accompagnées de l'expulsion accélérée des résidants du quartier. 7500 familles ont été ainsi déplacées. Cependant, de nombreux résidants ne peuvent pas déménager faute d'avoir les moyens d'acquérir un logement neuf et restent dans leur logement malgré leur insalubrité : dans les années 1950, les logements anciens, dont les courées, ont été confisqués par le gouvernement communiste et, aujourd'hui, ces logements appartiennent toujours pour la plupart à l'État et leurs occupants en sont encore locataires et non pas propriétaires; ils ne sont donc pas motivés à rénover ces vieux bâtiments, dont l'aspect délabré les marginalise encore un peu plus dans le quartier.

22 Les nouvelles opérations réalisées à l'échelle de vastes îlots regroupent en général un ensemble résidentiel sous forme de tours implantées en retrait de la rue et un centre commercial à l'alignement de la rue. La répartition des fonctions urbaines reste cependant relativement similaire d'une époque à l'autre, même si leurs traductions architecturales sont très différentes: une couronne commerciale, plus ou moins 
continue suivant le type urbain et architectural référent, qui protège les fonctions résidentielles tournées vers l'intérieur de l'îlot et dont l'accès est contrôlé.

Illustration 5

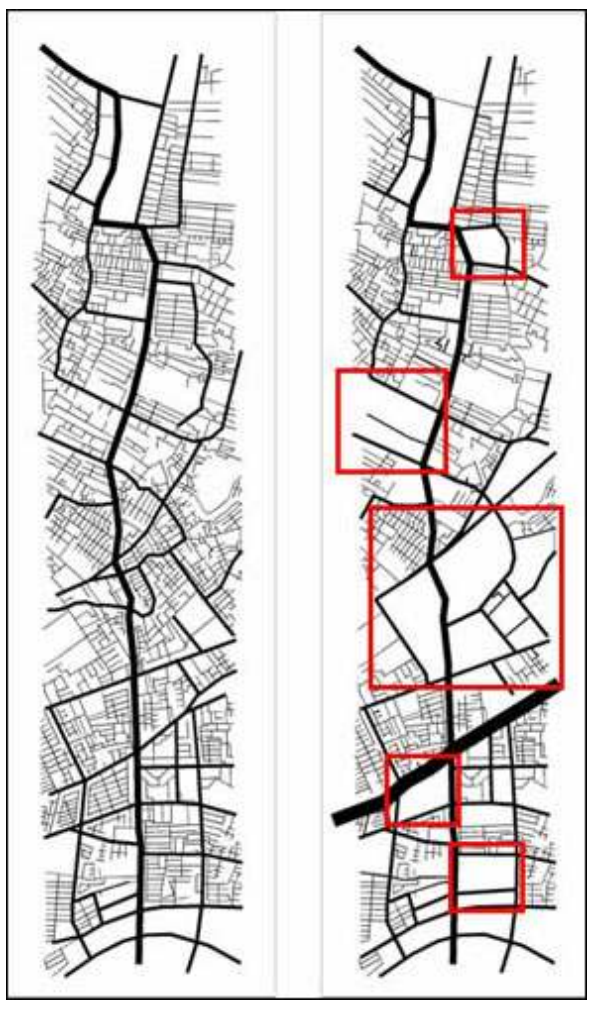

Photographie 6 - Nouvelles opérations rue du Sichuan

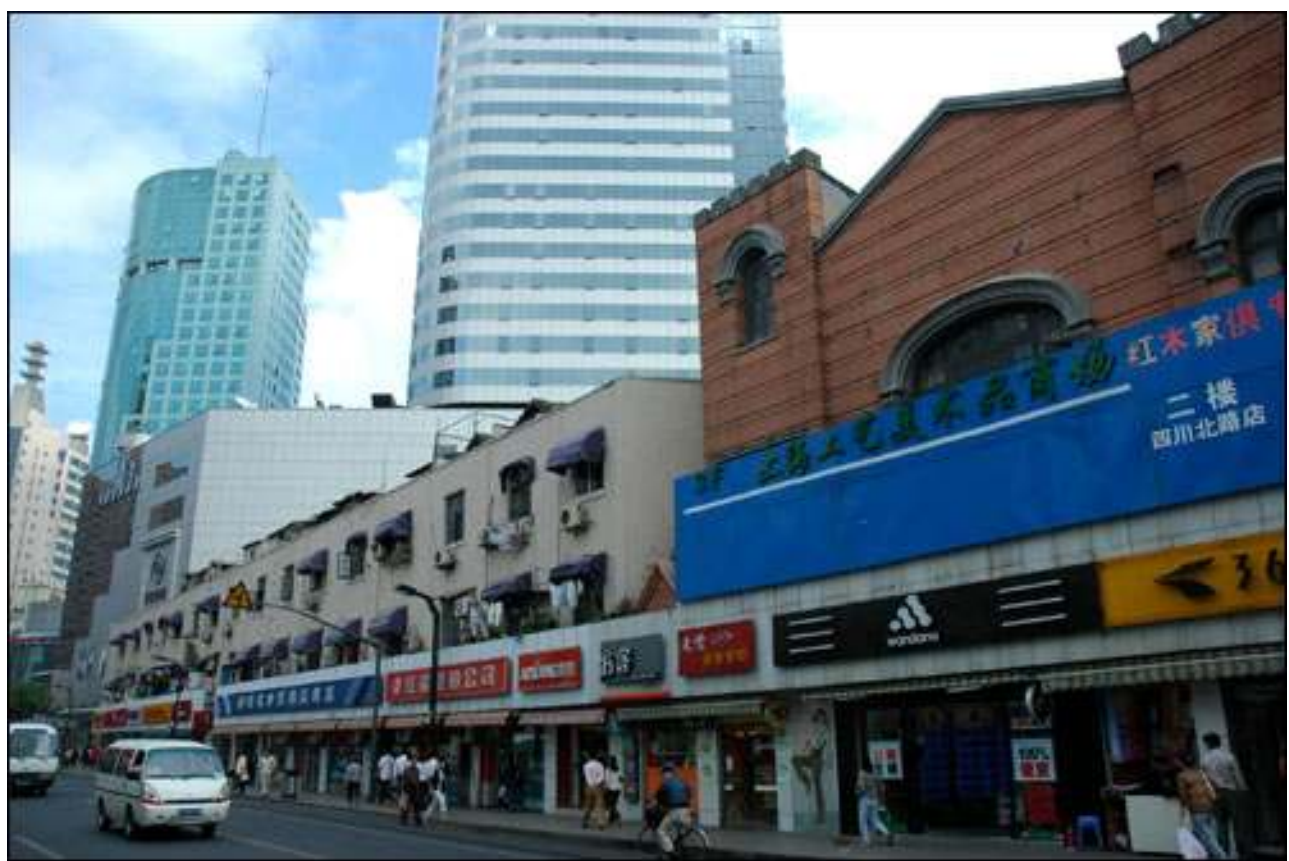

L'occupation résidentielle de la rue du faubourg Saint-Antoine n'a pas connu un tel bouleversement. Sa mutation s'est faite progressivement à la suite de différents 
phénomènes qui se sont conjugués dès les années 1970-90: déclin de l'artisanat du meuble ; politique de rééquilibrage de l'Est parisien menée par les différents maires de la capitale ; abandon « de la démolition-reconstruction pour la réhabilitation dans le souci de préservation du patrimoine " (Clerval A., 2009); "effet collatéral » de la politique de grands travaux qui a conduit à la construction de l'opéra Bastille dans les années 1990.

Les changements sociaux et économiques de ce faubourg, auparavant populaire, se traduisent donc de la même façon par un phénomène de gentrification, L'organisation de la mixité au sein de la parcelle s'est en même temps pour partie inversée. Historiquement étaient privilégiés au sein de l'immeuble sur rue le commerce à rez-dechaussée et les logements en étage, tandis que les fonctions de production et de stockage étaient regroupées en fond de parcelle autour de la cour de service. Aujourd'hui, avec la quasi disparition de l'artisanat, la gentrification résidentielle se traduit parfois par une forme d'inversion de l'occupation du bâti entre rue et cour : les locaux artisanaux sur cours sont transformés en «lofts " à vocation résidentielle ou d'activités de création, tandis que les activités tertiaires occupent plutôt l'immeuble sur rue.

\section{Les mobilités « actives » à l'épreuve de l'automobile}

Compte tenu de leur situation péricentrale, les deux rues se présentent comme des axes majeurs de déplacement à l'échelle intra-urbaine et de desserte locale en rapport avec leur vocation résidentielle, économique et commerciale: la rue du Sichuan (Nord) connecte le secteur historique du Bund au sud avec les périphériques intérieure et intermédiaire au nord, et irrigue le district de Hongkou, tandis que la rue du faubourg Saint-Antoine dessert les $\mathrm{XI}^{\mathrm{e}}$ et XII ${ }^{\mathrm{e}}$ arrondissements, en même temps qu'elle assure la liaison entre les pôles de la Bastille et de la Nation, et au-delà avec la porte de Vincennes, les boulevards des maréchaux et le périphérique.

Cette double fonction de déplacement génère un trafic automobile très important, en même temps qu'une fréquentation piétonnière considérable (plus de 100000 déplacements et 50000 traversées à pied par jour en 2003) et cycliste. Elle fait également de la rue du faubourg Saint-Antoine l'un des axes les plus accidentogènes de Paris, en raison de sa morphologie héritée.

Elle bénéficie aussi de la présence de quatre stations de la ligne 1 du métro, qui donne accès aux principaux pôles métropolitains de la rive droite - Châtelet, Louvre, ChampsÉlysées, Étoile...-, à chacune de ses extrémités - Bastille et Nation avec une correspondance avec le RER A -, et au droit des deux principaux carrefours - LedruRollin et Faidherbe-Chaligny.

Elle est par ailleurs desservie par une ligne de bus qui l'emprunte sur toute sa longueur, et deux lignes de bus qui la croisent au droit des deux mêmes principaux carrefours. Elle est donc totalement intégrée au réseau de transport en commun parisien et métropolitain, qui permet à l'usager de la rue de disposer d'une station à moins de 5 minutes à pied quelle que soit sa situation dans la rue.

29 Cependant, le profil en entonnoir de la rue, dont l'emprise passe d'environ $30 \mathrm{~m}$ à son débouché sur la place de la Nation à $17 \mathrm{~m}$ à son débouché sur la place de la Bastille avec un élargissement ponctuel à presque $40 \mathrm{~m}$ devant l'hôpital Saint-Antoine -, pose 
des problèmes de confort et de sécurité pour les modes de déplacement alternatifs à l'automobile - piéton, cycliste, bus - qui empruntent la rue.

Malgré les aménagements dissuasifs réalisés pour maîtriser la circulation et le stationnement automobile - une seule file de stationnement latéral en section la plus étroite -, les pratiques illicites de livraison et de stationnement rendent très difficiles la progression des bus et la traversée des piétons. Des aménagements sont actuellement à l'étude pour réduire les nuisances dues à la circulation automobile et assurer la priorité des transports en commun et garantir le confort des piétons. La concurrence entre piétons et automobilistes est clairement arbitrée par les pouvoirs publics en faveur des premiers.

31 La rue du Sichuan (Nord) est aujourd'hui victime de l'essor exponentiel de la motorisation des ménages, en particulier dans les grandes métropoles comme Shanghai, et qui se fait au détriment des pratiques cyclistes dominantes jusqu'aux années 1980. Ainsi en 2004 les déplacements non motorisés passaient sous la barre des $60 \%$ des déplacements à Shanghai, tandis que les déplacements en véhicule particulier atteignaient pour la première fois les $20 \%$. En réaction à cette évolution peu " vertueuse " par rapport aux exigences du développement durable, la Municipalité de Shanghai a mis en place un programme très ambitieux de développement de transports en commun lourds, notamment de lignes de métro, dans la perspective de l'exposition universelle de 2010. La rue du Sichuan (Nord) n'en bénéficie que ponctuellement avec une station sur son tracé, à son extrémité nord au droit du stade de football de Hongkou, et deux dans sa périphérie immédiate sur les rues parallèles, Hailun et Baoxing (Est). L'élargissement de la rue dans sa section sud a permis l'aménagement de larges trottoirs et une certaine fluidité du trafic automobile, mais a paradoxalement découragé la déambulation des piétons.

\section{Les pratiques commerciales, symptôme des mutations urbaines}




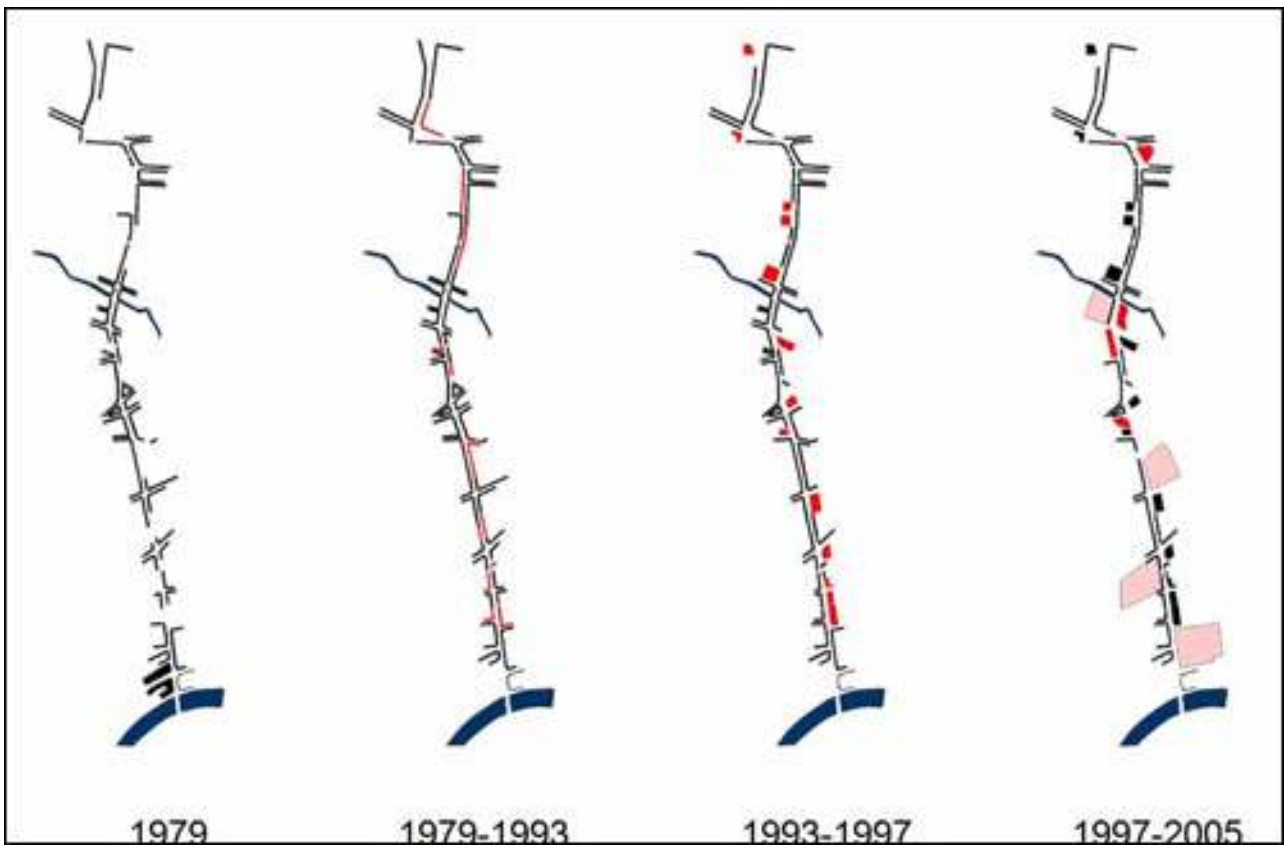

Les deux rues connaissent une mutation semblable du commerce qui d'indépendant devient pour beaucoup franchisé, en particulier dans l'habillement (Nike, Gap, Etam...) et la restauration rapide avec l'installation rue du Sichuan (Nord) de Pizza Hut, KFC et Starbucks Coffee, et de MacDonald's rue du faubourg Saint-Antoine. Le commerce alimentaire et les grandes surfaces différencient cependant les deux rues : la grande distribution est présente rue du faubourg Saint-Antoine sous forme de supérettes ou de moyennes surfaces (Monoprix, Auchan, Ed...), mais on ne trouve pas de centre commercial, au contraire de la rue du Sichuan (Nord), où se sont ouverts plusieurs grands complexes commerciaux, tandis que le commerce alimentaire se repliait sur les voies locales qui bordent les autres façades des îlots riverains. 
Photographie 7 - Commerces, rue du faubourg Saint-Antoine

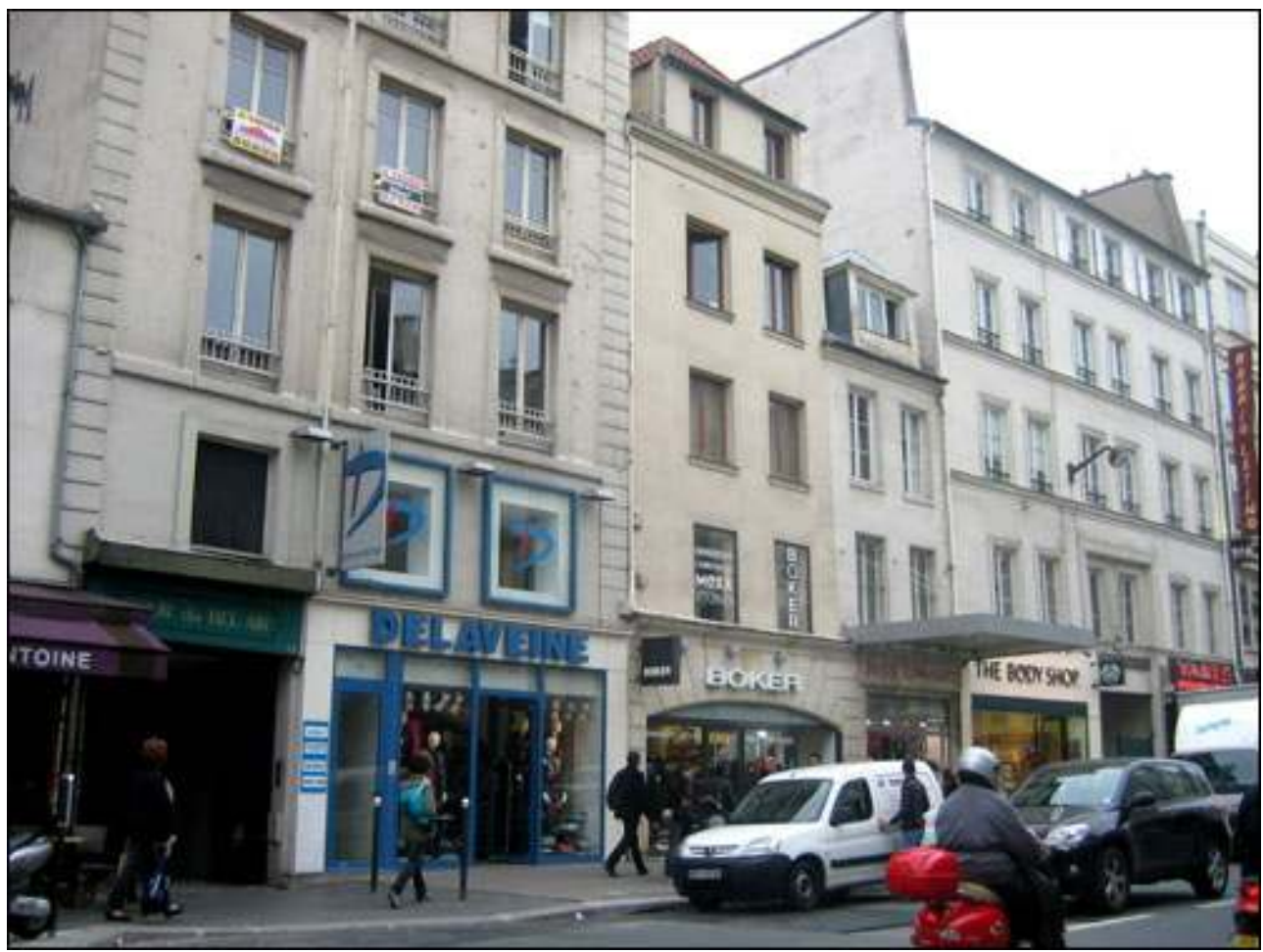

Photographie 8 - Commerces, rue du Sichuan

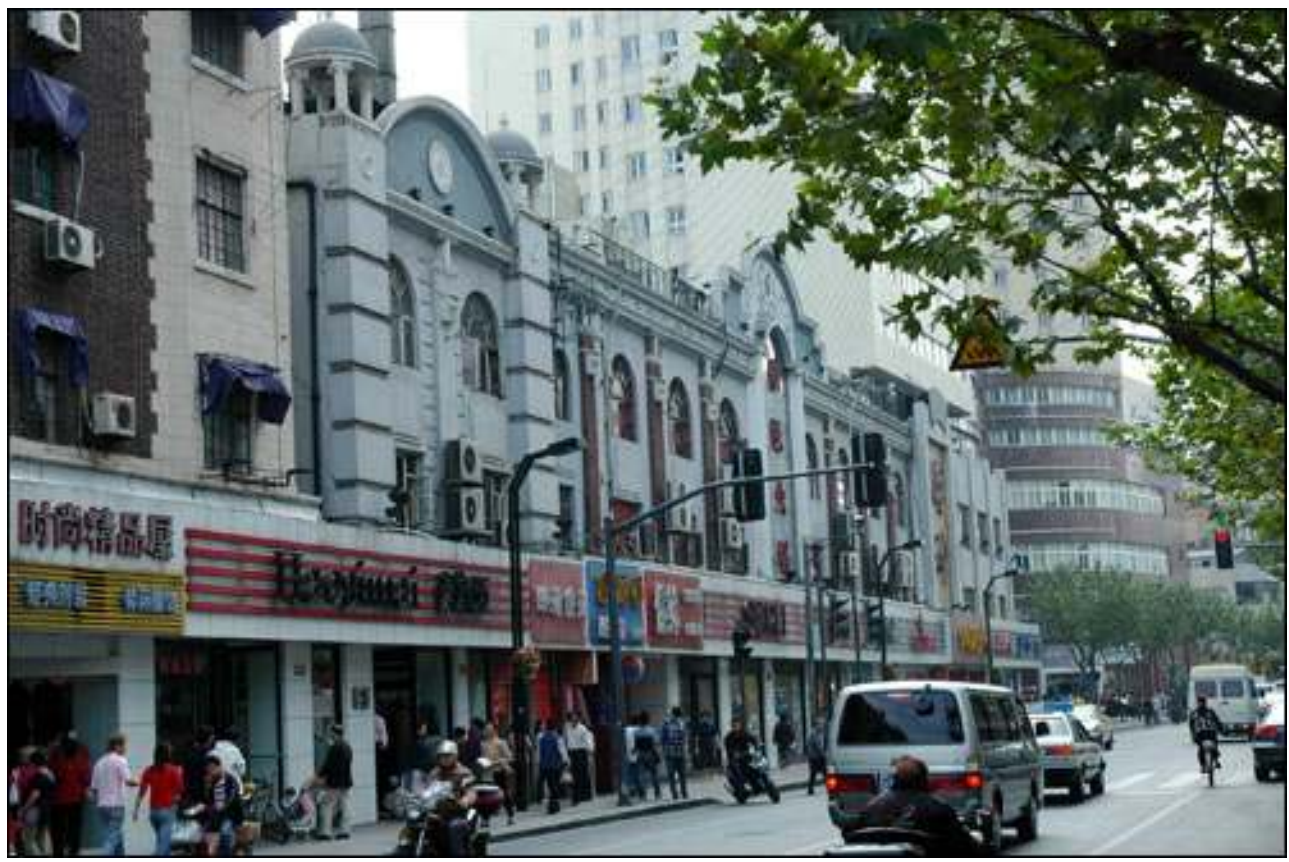

Les observations et les enquêtes portant sur les pratiques urbaines de la rue du Sichuan (Nord) ont révélé un effet générationnel très fort qui tient à la rupture profonde dans les usages résidentiels et de mobilité des différentes tranches d'âge, et qui se concrétise dans leurs pratiques commerciales très spécifiques. Jusqu'à l'époque de « l'ouverture et des réformes ", l'unité de travail (danwei) fonctionnait comme unité de lieu de vie, qui regroupait toutes les fonctions urbaines et les organisait dans un rapport de proximité, 
tandis que les déplacements étaient contrôlés à travers le livret d'enregistrement civil (hukou). Aujourd'hui, le mode de vie "globalisé » des jeunes générations post-réformes les conduit à occuper l'espace urbain en toute liberté et à déambuler dans la rue en combinant pratiques d'achat, de restauration et de loisirs.

Les résidants d'âge mûr qui ont connu l'époque des réformes se différencient dans leurs pratiques urbaines suivant qu'ils sont arrivés récemment dans le quartier dans le cadre des opérations de rénovation ou de privatisation des logements publics ou qu'ils sont là de longue date. Ces derniers, qui n'ont pas eu la possibilité d'aller résider ailleurs, vivent comme une contrainte la transformation de leur cadre de vie et se sentent rejetés de l'espace public par ses usagers plus jeunes et/ou plus riches. De la même manière que les résidants plus âgés, les retraités de l'époque maoïste, ils compensent cette désappropriation contrainte de l'espace public, soit en trouvant refuge au cœur de leur îlot de vie, soit en reportant leur sociabilité dans les parcs à la faveur des activités de plein air propres à la culture urbaine chinoise (gymnastique, danse, chant, jeux...).

Les enquêtes menées rue du faubourg Saint-Antoine ne révèlent pas une telle rupture dans les pratiques de l'espace public, qu'elles soient résidentielles ou commerciales. Les différences générationnelles semblent beaucoup moins marquées, et les transformations sociales sont nettement plus progressives. Ainsi peut-on affirmer que « la différence de pratiques de la rue est donc plus importante entre les Chinois jeunes et âgés qu'entre les jeunes chinois et français » (Perchet F., 2008).

\section{Gentrification et morphologie : un double processus de rupture dans les usages de la rue chinoise}

À partir des concepts proposés par Catherine Bidou-Sachariasen (2003), l'analyse de ces deux rues permet d'observer un double processus de gentrification de l'espace public: une gentrification qui concerne les résidents riverains de la rue, qu'on pourrait qualifier d' "occupation"; et une gentrification qui concerne les pratiques de consommation des usagers de la rue, qu'ils soient habitants du quartier ou simplement passants et/ou chalands, qu'on pourrait qualifier de "fréquentation», et qui "par le changement d'image du quartier et de la rue qu'elles engendrent, favorisent certaines transformations de l'espace social» (Fleury A., 2004). Ce double processus de gentrification se développe de façon très différente dans les deux rues étudiées ici.

Comme déjà évoqué, la rue du faubourg Saint-Antoine connaît depuis plusieurs décennies un lent processus de gentrification résidentielle. Il s'apparente à ce qu'a pu observer David Ley dans les métropoles nord-américaines (Ley D., 1996), avec une anticipation du processus par les professions artistiques, confortée dans le cas présent par les "grands projets » d'État à vocation culturelle réalisés au même moment dans son environnement (opéra Bastille, viaduc des Arts). Cette gentrification résidentielle a généré à la suite une certaine forme de gentrification de consommation.

La mutation récente des pratiques de la rue du Sichuan (Nord) s'appuie avant tout sur une gentrification de fréquentation, encouragée par une politique très ambitieuse de renforcement de sa vocation commerciale métropolitaine à travers la réalisation de grands centres commerciaux. On est confronté ici à une stratégie urbaine globale, portée par le gouvernement local de concert avec les investisseurs privés, et dont la 
généralisation géographique est pointée par Neil Smith " de Sao-Paulo à Puebla... et de Shanghai à Séoul » (Smith N., 2002). Sous couvert de "régénération urbaine ", il s'agit avant tout d'insérer la ville dans l'économie globale, en inscrivant dans son espace physique et social les nouvelles fonctions et formes urbaines qui la caractérisent. Les mutations d'occupation n'ont fait qu'aggraver cette rupture dans l'espace social.

Photographie 9 - Centres commerciaux, rue du Sichuan

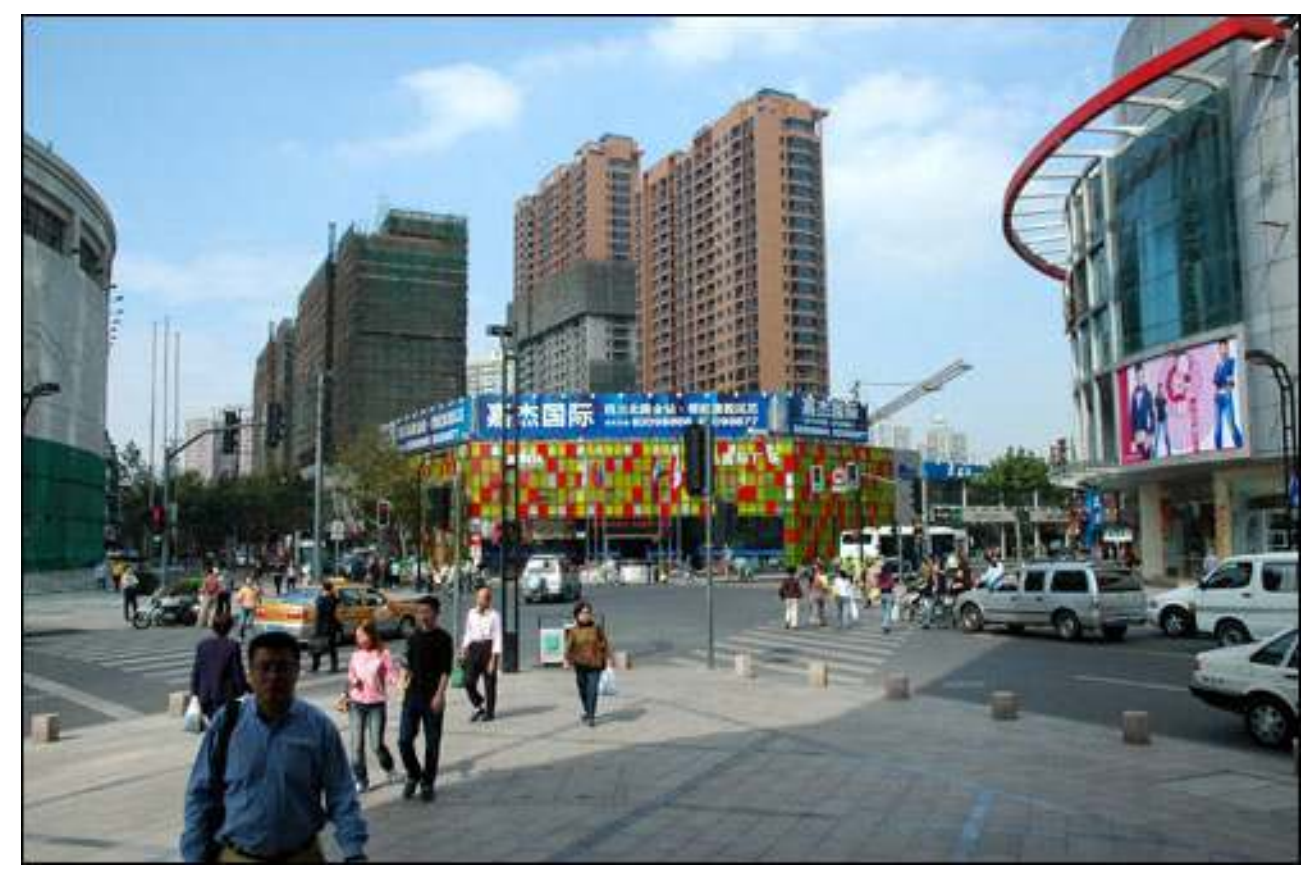

Photographie 10 - Commerce, rue du Sichuan

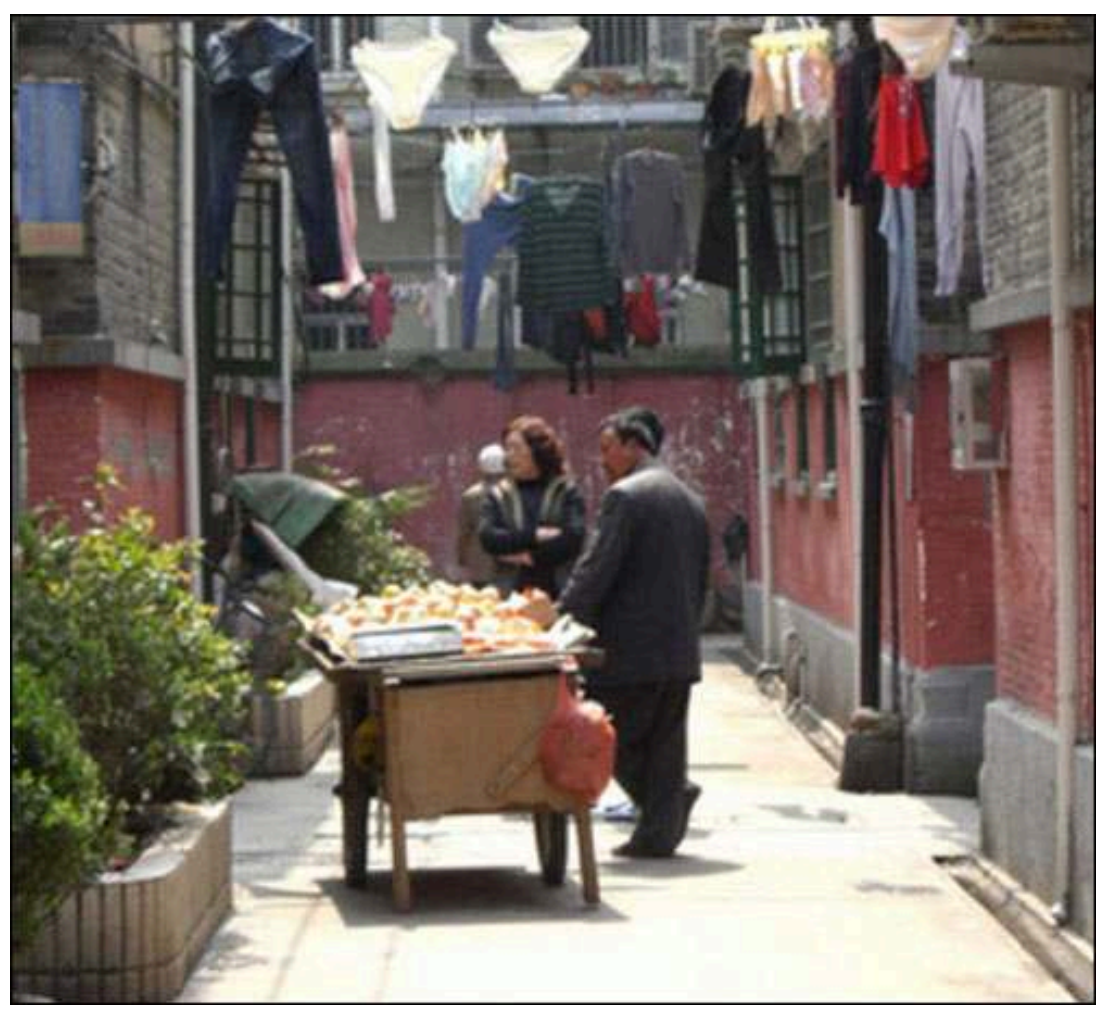


étudiées ici sont considérables. Ils tiennent bien évidemment à des modalités et des échelles d'intervention sur la ville et des statuts du foncier très différents. On observe notamment rue du faubourg Saint-Antoine, et plus globalement à Paris, une grande inertie morphologique et d'occupation des territoires de la voie, qui trouve son origine dans la taille des îlots, leur morcellement en petites unités, les parcelles -qui n'ont pas vraiment leur équivalent en Chine, dont le foncier a été collectivisé dans les années 1950 - et dont la propriété est elle-même diluée entre petits propriétaires privés ou copropriétaires de locaux à usage résidentiel mais aussi commercial, ce qui intensifie la mixité de la rue en pérennisant l'occupation des espaces riverains.

On ne saurait trop insister avec Jane Jacobs sur le rôle du temps dans le processus de renouvellement urbain, qui permet d'alterner et de combiner renouvellement de la forme et renouvellement de l'usage. La rue du Sichuan (Nord), et plus généralement la rue chinoise, connait depuis plus vingt ans des bouleversements considérables qui ont rompu ce processus continu de transformation de ses espaces riverains et des pratiques qu'ils accueillent, et qui en ont gommé pour une bonne part ses singularités sociales et morphologiques. Pour paraphraser J.-L. Gourdon cité en introduction, la rue chinoise, en particulier shanghaienne, en raison des destructions qu'elle a subies, n'a pu que rarement préserver son unité dans la diversité et le changement.

\section{BIBLIOGRAPHIE}

Brès A., 2007. De la voirie à la rue : riveraineté et attrition. Des stratégies d'inscription territoriale des mobilités. Flux, n 66-67.

Bidou-Sachariasen C., dir., 2003. Retours en ville. Paris, Descartes.

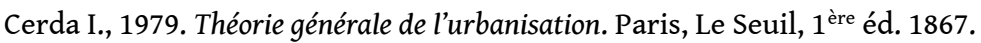

EchoGéo, 12 | 2010 
Clerval A., 2009. Les politiques publiques face à la gentrification. Le cas de Paris intra-muros. In Pérennité urbaine ou la ville par-delà ses métamorphoses, t. 2 Turbulences, Colette Vallat, Aurélien Delpirou et Fabrizio Maccaglia (dir.), Paris, L'Harmattan, p. 139-151.

Durant S., Giacomoni A., Lançois C., 2007. Les interactions entre l'établissement et le mouvement, exemple de la rue du faubourg Saint-Antoine. Paris, mémoire de master 2, Université Paris 1.

Fleury A., 2004. La rue, un objet géographique. Tracés, Revue de Sciences humaines, $n^{\circ} 5$.

Ged F., 2000. Shanghai. Paris, IFA.

Gourdon J.-L., 2001. La rue. Essai sur l'économie de la forme urbaine. Paris, L'Aube.

Jacobs J.,1991. Déclin et survie des grandes villes américaines. Paris, Mardaga, $1^{\text {ère }}$ éd. 1961.

Ley D., 2003. Artists, aestheticisation and the field of gentrification. Urban Studies, $\mathrm{n}^{\circ} 12$

Paquot T., 2009. L'espace public. Paris, La Découverte.

Paris - Projet, Quartiers anciens, Approches nouvelles, 1998, n³2-33.

Perchet F., 2008. Évolution des usages de la rue dans le cadre du processus de gentrification des villes. Paris, mémoire de master 1, Université Paris 1.

Sanjuan T., 2006. Dictionnaire de la Chine contemporaine. Paris, Armand Colin.

Sanjuan T., 2009. Atlas de Shanghai. Paris, Autrement.

Smith N., 1996. The New Urban Frontier : gentrification and the revengist city. Routledge.

\section{RÉSUMÉS}

Alors que les métropoles chinoises connaissent une globalisation accélérée, l'observation de deux rues, l'une chinoise, l'autre parisienne, aide à comprendre ce qui fait leur permanence et leur singularité, notamment les différences et les similitudes qui concernent les différents aspects de leur mutation. Ce sont en particulier les caractéristiques du processus de gentrification que ces deux rues connaissent qui les différencient: pour la rue du faubourg Saint Antoine domine une gentrification résidentielle, de type socio-culturelle, qui s'affirme depuis une trentaine d'année; tandis que pour la rue Sichuan (Nord), on observe une gentrification de fréquentation, encouragée par une politique très volontaire de renforcement rapide de sa vocation commerciale à l'échelle métropolitaine au détriment des espaces et des populations riveraines.

\section{INDEX}

Mots-clés : Chine, commerce, gentrification, riveraineté, rue

Keywords : China, commercial activity, gentrification, street, streetside relationship

\section{AUTEURS}

\section{ANTOINE BRÈS}

Antoine Brès, architecte urbaniste, associé de l'agence Brès+Mariolle et associés, enseigne également à l'Université de Paris 1 Panthéon-Sorbonne. Ses dernières publications : 
- Vitesse et Urbanisme, l'enjeu de la riveraineté, 2009. In Chronoaménagement et Autoroute autrement, sous la direction de SMSD (Syndicat Mixte du Schéma Directeur) et AURG (Agence d'Urbanisme de la Région de Grenobloise), Editions du CERTU, 9 p.

- De la ville de la courte distance à la ville polycentrique : densifier à partir des gares, septembre 2009. Avec Béatrice Mariolle, Transports urbains, Mobilités-Réseaux-Territoires $n^{\circ} 115,5$ p.

- L'urbain, une halte plus ou moins étendue et complexe, 2008. In La mobilité qui fait la ville, sous la direction de Yves Chalas et Florence Paulhiac, Editions du CERTU, 22 p.

antoinebres@bresmariolle.fr

\section{JEAN-FRANÇOIS CUENOT}

Jean-Francois Cuenot est Ingénieur d'études CNRS à l'UMR PRODIG. jfcuenot@univ-paris1.fr

\section{THIERRY SANJUAN}

Thierry Sanjuan est Professeur de géographie à l'Université Paris 1 Panthéon-Sorbonne et directeur de l'UMR PRODIG. tsanjuan@univ-paris1.fr 